\title{
Timanoplastia Tipo 1 e Miringoplastia na Residência - Resultados Cirúrgicos e Audiométricos
}

\section{Timanoplastia Myringoplasty Type 1 and in Residency Surgical Results and Audiometric}

\author{
Edgar Sirena*, Bettina Carvalbo*, Mauricio Buscble**, Marcos Mocellin***.
}

\author{
* Graduação em Medicina. Médico (a) Residente da Otorrinolaringologia no HC/UFPR. \\ *** Mestre em Medicina (Clínica Cirúrgica) pela Universidade Federal do Paraná, Brasil (1999). Professor Adjunto da Disciplina de Otorrinolaringologia da UFPR - Universidade \\ Federal do Paraná, Brasil. \\ *** Doutor em Otorrinolaringologia pela Escola Paulista de Medicina, Brasil (1986). Professor Titular da Universidade Federal do Paraná, Brasil. \\ Insitituição: Hospital de Clínicas da Universidade Federal do Paraná HC/UFPR \\ Curitiba / PR - Brasil. \\ Endereço para correspondência: Hospital de Clínicas da UFPR - Av. General Carneiro, 181 - Curitiba / PR - Brasil - CEP: 80060-900 - Telefone: (+55 41) 3360-1800 \\ -E-mail: cenf@hc.ufpr.br \\ Artigo recebido em 11 de Julho de 2010. Artigo aprovado em 22 de Agosto de 2010.
}

\section{RESUMO}

Introdução:

A timpanoplastia tem por objetivo reconstruir a membrana timpânica, restaurando a proteção à orelha media e melhorando a audição. Nesse estudo foram avaliados os resultados cirúrgicos e audiométricos dessa cirurgia, realizada no serviço de Otorrinolaringologia do HC/UFPR por residentes do 20 ano durante o ano de 2008, bem como alguns fatores que possam influenciar nos resultados.

Método: Estudo retrospectivo, através de analise de prontuários.

Resultados: Dos 31 pacientes avaliados, houve fechamento da perfuração em 24 (80\%) e melhora da audição com redução ou fechamento do gap condutivo em $60 \%$ e $26,7 \%$ respectivamente.

Discussão: A taxa de sucesso da cirurgia foi satisfatória, sendo similar à encontrada na literatura, sendo que fatores como idade, presença de patologia uni ou bilateral e tamanho da perfuração não foram fatores determinantes deste sucesso cirúrgico.

Conclusão: A timpanoplastia realizada por residentes do 20 ano da residência mostrou resultados satisfatórios tanto cirúrgicos quanto audiométricos.

Palavras-chave: audiometria, resultado de tratamento, timpanoplastia, membrana timpânica.

\section{SUMMARY}

Introduction:

The tympanoplasty aims to reconstruct the tympanic membrane, restoring protection to the middle ear and improve hearing. In this study we evaluated the surgical results and audiometric this surgery, performed in the service of Otorhinolaryngology, HC / UFPR by residents of the second year in the year 2008 and factors that may influence the results.

Method: A retrospective study through review of medical records.

Results:

Discussion: Among the 31 patients evaluated, there was closure of the perforation in $24(80 \%)$ and hearing improvement with reduction or closure of the conductive gap by $60 \%$ and $26.7 \%$ respectively. The success rate of surgery was satisfactory and similar to that found in the literature, and factors such as age, presence of unilateral or bilateral pathology and size of perforation were not determinants of surgical success.

Conclusion: Tympanoplasty performed by residents of the second year of residence showed satisfactory results regarding both surgical audiometric.

Keywords: audiometry, treatment outcome, tympanoplasty, tympanic membrane. 


\section{INTRODUÇÃO}

A otite média (OM) é uma doença mundialmente prevalente. Apesar de todos os avanços científicos (antibioticoterapia, tecnologia e conhecimento), a OM continua sendo considerada como um importante problema de saúde pública, uma vez que a otalgia, o desconforto, a perda auditiva, a otorreia, o trauma psicológico e as complicações causam grande sofrimento pessoal e familiar $(1,3)$.

A otite média crônica é caracterizada clinicamente como uma condição inflamatória associada a perfurações persistentes da membrana timpânica e otorreia. Histologicamente pode ser definida como um processo inflamatório da orelha média associada a alterações teciduais irreversível. Podem ser subdividida em otite média crônica não-colesteatomatosa (OMCNC) e otite média crônica colesteatomatosa (OMCC). A diferença entre esses dois grupos está na presença ou ausência do colesteatoma $(1,3)$.

O principal sintoma apresentado pelos pacientes acometidos pela OMCNC simples é a otorreia intermitente, normalmente associada a episódios de infecções de via aérea superior ou história de contaminação extrínseca (banho de piscina, mar), indolor e inodora, acompanhada de perda auditiva. À otoscopia geralmente encontramos uma perfuração na parte tensa da membrana timpânica de tamanho e forma variados, sendo a mucosa da orelha média com aparência quase normal, exceto por alguns graus de hiperemia $(1,3)$.

A membrana timpânica (MT) é formada por três camadas: uma camada externa, composta por epitélio escamoso queratinizado, uma camada fibrosa mesodérmica intermediária e uma camada mucosa endodérmica interna. Quando em solução de continuidade, a MT se regenera a partir de dois mecanismos de migração epitelial conhecidos. Um deles é o movimento centrífugo a partir do umbigo da MT. O segundo padrão mitótico, considerado essencial para a cicatrização das soluções de continuidade, é o movimento centrípeto, que ocorre em toda pars tensa da MT, com maior atividade ao redor do anel timpânico. Nas miringoplastias, o enxerto funciona como um substituto do extrato córneo sobre o qual deslizam correntes de migração epitelial, a fim de reparar a perfuração $(2,4)$.

A timpanoplastia tipo 1 e a miringoplastia, cirurgias realizadas para OMCNC simples, têm como objetivo a reconstrução da membrana timpânica, o restabelecimento da proteção sonora à janela redonda através da obtenção de uma cavidade preenchida por ar e restauração dos mecanismos que conduzem o som, melhorando a audição e cessando a otorreia (5).
Nesse estudo avaliamos os resultados cirúrgicos e audiométricos dessas cirurgias em nosso serviço no ano de 2008, por residentes do segundo ano, sob supervisão de um mesmo preceptor (M.B.), bem como alguns fatores que possam influenciar nos resultados.

\section{MÉTODO}

Foram incluídos nesse trabalho 30 orelhas de 21 pacientes portadores de OMCNC simples, submetidos à timpanoplastia tipo 1 e miringoplastia no Hospital de Clínicas de Curitiba no ano de 2008, por residentes do segundo ano e sob supervisão de um mesmo preceptor (M.B.).

O estudo realizado foi retrospectivo, com base em revisão de prontuários e foi aprovado pelo Comitê de Ética em Pesquisa em Seres Humanos do Hospital de Clínicas / UFPR. Os pacientes foram avaliados previamente a cirurgia por residentes e pelo mesmo orientador (M.B.), através de anamnese dirigida, exame otorrinolaringológico e exame audiométrico.

$\mathrm{Na}$ anamnese, os pacientes foram interrogados quanto ao início dos sintomas, história de otorreia, período sem otorreia, controle desta com gota otológica e cuidados para não molhar a orelha, associação com infecção de vias aéreas superiores, além de história de cirurgia otológica prévia e queixas nasais. À otoscopia, foram avaliadas as características, tamanho e posição da perfuração, desenhando-a no prontuário, além da complementação do exame otorrinolaringológico. Todos os pacientes foram submetidos à audiometria tonal e vocal previamente a cirurgia.

As técnicas cirúrgicas utilizadas foram a "in-lay" para perfurações pequenas e a "underlay" com acesso retroauricular para os demais tipos de perfurações. Quanto ao tipo de enxerto, utilizou-se fáscia temporal ou aureolar para a técnica "underlay" e cartilagem com pericôndrio do trago para a técnica "in-lay".

A técnica "underlay" por via retroauricular consistia na realização de: 1) infiltração com solução de xilocaína + adrenalina 1: 50.000 na região retroauricular e meato auditivo externo; 2) confecção dos retalhos de Lempert I, II e III por via transmeatal (Lempert I: incisão inicia no anel timpânico, abrangendo pele e periósteo e extende-se para fora, ao longo da junção das paredes superior e posterior do meato auditivo externo, ultrapassando o apex do triangulo supra-meatal. Lempert II: a incisão começa no anel timpânico, acompanha a junção das paredes posterior e inferior do meato auditivo externo e termina na porção inferior da borda anterior da concha. Lempert III: a incisão 
acompanha a margem timpânica da parede posterior do meato, abrangendo pele e periósteo, unindo as duas primeiras incisões. (6)) 3) incisão retroauricular da pele e tecido subcutâneo, com retirada do enxerto de fáscia aureolar ou temporal; 4) confecção do retalho periosteal e descolamento até encontrar as incisões no meato acústico externo; (5) avivamento das bordas da perfuração com retirada de placas de timpanosclerose; 6) descolamento e rebatimento do retalho timpanomeatal (conforme a posição e o tamanho da perfuração, a incisão no meato auditivo é ampliada anteriormente e é descolado um retalho timpanomeatal do ligamento maleolar posterior ao ligamento maleolar anterior); 7) Revisão da cadeia ossicular; 8) Colocação do enxerto de fáscia sob o cabo do martelo e sobre a bigorna; 9) Reposicionamento do retalho timpanomeatal; 10) Se necessário, colocação de "gelfoam" por baixo do enxerto; 11) Colocação de "gelfoam" pressionando o retalho timpanomeatal sobre o meato auditivo externo; 12) sutura por planos; 13) curativo externo.

A técnica "in-lay" foi realizada através de: (1) escarificação das bordas da membrana timpânica por via transmeática; (2) confecção de enxerto com cartilagem e pericôndrio de trago, formando um carretel; (3) colocação do enxerto, da mesma maneira que se coloca um tubo de ventilação.

O acompanhamento pós-operatório foi com retornos iniciais em 2, 7, 30, 60 e 90 dias e retornos posteriores periódicos. Os exames audiométricos foram realizados entre 90 e 180 dias de pós-operatório.

\section{RESULTADOS}

Foram submetidos à timpanoplastia 30 orelhas de 21 pacientes com otite média crônica simples, sendo 16 (76\%) do sexo feminino e 5 (24\%) do sexo masculino, com média de idade de 30,1 anos, variando de 7 a 64 anos.

Dos 21 pacientes, 13 (62\%) tinham patologia bilateral e 8 (38\%) unilateral. Entre os que tinham perfuração bilateral, 8 foram submetidos à timpanoplastia nas 2 orelhas em tempos diferentes em 2008 e ambos incluídos no trabalho. Em apenas 1 caso a cirurgia foi realizada nas 2 orelhas no mesmo tempo cirúrgico, um com técnica "underlay" retroauricular e o outro com técnica "in-lay" com enxerto de cartilagem do trago. Observando o resultado cirúrgico, das 22 orelhas operadas quando havia patologia bilateral, $18(81,8 \%)$ apresentaram fechamento da perfuração e dos 8 com patologia unilateral, 6 (75\%) tiveram o mesmo resultado.

Das 30 orelhas operados, $20(66,7 \%)$ foi à orelha esquerda e $10(33,3 \%)$ a orelha direita. Com relação ao resultado cirúrgico, 15 (75\%) das 20 orelhas esquerdas e 9 (90\%) das 10 orelhas direitas operadas tiveram fechamento da perfuração.

Com relação à localização e tamanho da perfuração, 12 (40\%) orelhas tinham perfuração ampla, ou seja, que atingia grande parte da pars tensa da membrana timpânica, acometendo quadrante posteriores e anteriores, 12 (40\%) tinham perfurações localizadas em quadrantes posteriores e 6 (20\%) apresentavam perfurações em quadrantes anteriores. Dos 12 casos com perfurações amplas, 10 (83,3\%) tiveram fechamento da perfuração e $2(16,7 \%)$ mantiveram perfuração residual. Considerando as 12 orelhas com perfurações posteriores, 9 (75\%) fecharam a perfuração e $3(25 \%)$ não fecharam. E dos 6 casos com perfurações anteriores, 5 (83,3\%) apresentaram neotímpano íntegro no pós-operatório e $1(16,7 \%)$ manteve a perfuração.

A principal técnica utilizada foi a "underlay" por acesso retroauricular com enxerto de fáscia aureolar ou temporal em 28 ouvidos (93,3\%) e em 2 casos (6,6\%) onde a perfuração era pequena foi utilizada a técnica "in-lay" com enxerto de cartilagem e pericôndrio do trago.

O sucesso cirúrgico, considerando o fechamento da perfuração da membrana timpânica, foi de 24 orelhas (80\%). Em relação à avaliação audiométrica, a grande maioria dos pacientes teve melhora auditiva. Comparando a média de via aérea (mVA) das frequências 0,5, 1 e 2 KHz pré e pós-operatória de todos os ouvidos operados, observamos uma melhora média de $12,3 \mathrm{~dB}$. Se considerarmos somente as orelhas em que houve o fechamento da perfuração, a melhora média da mVA foi de $14,1 \mathrm{~dB}$. Com relação ao "gap" condutivo, em 8 casos $(26,7 \%)$ foi fechado, em 18 casos (60\%) reduziu, em 3 (10\%) permaneceu igual e em 1 caso (3,3\%) o "gap" aumentou. Nesse último caso houve fechamento da perfuração, porém a paciente fez uma otite média com efusão no pós-operatório que estava sendo tratada.

\section{DISCUSSÃO}

As primeiras cirurgias com o objetivos de fechamento de perfuração de membrana timpânica datam de 1878 descritas por BERTHOLD, sendo mais consolidadas a partir de 1952, por Wullstein (7).

Os principais objetivos da timpanoplastia são obtenção de uma orelha média seca, através de uma membrana timpânica íntegra, e uma melhora audiométrica. A taxa de sucesso na literatura apresenta uma ampla variação (8).

Em nosso estudo encontramos uma taxa de sucesso de $80 \%$, quando avaliamos o fechamento da perfuração, o 
que pode ser considerado um resultado satisfatório, tendo em vista a população sócio-econômica estudada e o fato das cirurgias terem sido realizadas por residentes do segundo ano. SHeehy (9) em uma revisão de 472 casos apresentou um índice de fechamento da perfuração de MT de $97 \%$, enquanto Котесна (10), em seu estudo, apresentou um índice de 82,2\%. Black (11) mostrou um sucesso cirúrgico de 66,6\%, e Pinar (12) encontrou 74,4\%. Bunzen (4) em uma avaliação de 97 orelhas submetidas à timpanoplastia, obteve neotímpano íntegro no pós-operatório em 80,4\% dos casos e Fukuchi (3) em estudo realizado com 37 pacientes na Faculdade de Medicina do ABC obteve 51, $4 \%$ de fechamento da perfuração da MT na primeira cirurgia e $65 \%$ considerando as re-operações.

A idade é um fator que não altera a taxa de sucesso nas timpanoplastias. Na população idosa deve-se fazer uma avaliação pré-anestésica mais rigorosa, além da realização de um cuidado pré-operatório, com avaliação do estado nutricional do paciente, estado cardiovascular, metabólico e as condições mentais (13). Já para a população pediátrica, deve-se atentar para o perfil psicológico da criança, que deve obedecer rigorosamente às medidas de repouso e proteção auricular, além da idade suficiente para um adequado desenvolvimento da mastoide, da tuba auditiva e da imunidade (14). A taxa de sucesso de timpanoplastia em criança provavelmente não é uma questão de idade, mas uma questão de seleção de pacientes. É considerado um procedimento seguro, com resultados anatômicos e funcionais comparáveis ao relatado para adultos $(15,16,17)$.

Na comparação dos resultados cirúrgicos quando os pacientes tinham patologia uni ou bilateral não encontramos diferença significativa entre os resultados, por serem valores próximos e dado o número relativamente pequeno de pacientes avaliados. Pinar (12) em um estudo com avaliação de 231 pacientes, mostrou que a orelha oposta saudável pode ser considerado um fator prognóstico independente para o sucesso cirúrgico.

Normalmente não realizamos timpanoplastia bilateral em um mesmo tempo cirúrgico em nosso serviço, com exceção de casos onde em pelo menos um dos lados é realizado a técnica "in-lay" com enxerto de cartilagem do trago.

Caye-Thomasen et al (18) em seu estudo, avaliaram 26 pacientes submetidos à miringoplastia bilateral em um mesmo tempo cirúrgico, sendo a maioria por via transcanal e técnica "onlay", apresentando bons resultados e boa aceitação por parte dos pacientes.

As perfurações localizadas nos quadrantes anteriores representam um acesso cirúrgico pior para se atingir à borda anterior, além de serem menos vascularizadas, o que as deixam com pior prognóstico. Para muitos autores, o local da perfuração é considerado um parâmetro mais importante do que o seu tamanho para o sucesso da cirurgia $(8,19)$. Nesse estudo, não encontramos diferença importante nos resultados cirúrgicos, quando comparamos os locais das perfurações, talvez pelo número relativamente pequeno de casos avaliados.

A grande maioria dos ouvidos operados apresentou melhora auditiva com diminuição ou fechamento do "gap" aéreo-ósseo, mesmo alguns que persistiram com perfuração residual. Em apenas 3 casos a audição se manteve igual, com o mesmo "gap," e em 1 caso houve aumento do "gap" aéreo-ósseo apesar do fechamento da perfuração, devido ao paciente ter feito otite média com efusão no pósoperatório, que está sendo resolvida.

Lateralização e "blunting" são complicações possíveis no pós-operatório da timpanoplastia. Ambas podem causar persistência de perda auditiva condutiva. A primeira acontece quando a superfície da membrana timpânica fica localizada lateral ao anel ósseo e perde o contato com os mecanismos de condução da orelha média (20). A segunda acontece quando ocorre uma perda do angulo agudo tímpano-meatal anterior, geralmente por falha na técnica cirúrgica ou fibrose durante a cicatrização (21). Em nosso estudo não encontramos nenhum caso de lateralização da membrana timpânica ou "blunting".

O sucesso cirúrgico encontrado em nosso estudo mostra resultados semelhantes a trabalhos anteriores realizados em nosso serviço. Coifman (22) em sua dissertação de mestrado em 1992 avaliou 101 pacientes submetidos à timpanoplastia no Hospital de Clínicas de Curitiba e encontrou uma taxa de fechamento da perfuração de membrana timpânica de 81,19\%.

\section{CONCLUSÃO}

Os resultados cirúrgicos e audiométricos obtidos nesse trabalho podem ser aceitos como satisfatórios e dentro do esperado pela literatura, tendo em vista que as cirurgias foram realizadas por Residentes do segundo ano e considerando também o nível sócio-econômico da população de estudo, que muitas vezes pode dificultar a aquisição dos medicamentos prescritos e os cuidados adequados no pós-operatório.

\section{REFERÊNCIAS BIBLIOGRÁFICAS}

1. Costa SS, Sousa LCA. Otite média crônica nãocolesteatomatosa. In: Campos CAH, Costa HOO. Tratado de Otorrinolaringologia. São Paulo: Roca; 2002. p. 72-92. 
2. Costa S, Cruz OLM, Kluwe LHS, Smith MM. Timpanoplastias. Em: Cruz, OLM e Costa, S (eds). Otologia clínica e cirúrgica. 1ª ed. São Paulo: Editora Revinter; 1980, pp. 245-271.

3. Fukuchi I, Cechiari DP, Garcia E, Rezende CEB, Rapoport PB. Timpanoplastias: resultados cirúrgicos e análise dos fatores que podem interferir no seu sucesso. Rev Bras Otorrinolaringol. 2006, 72(2):261-6.

4. Bunzen D, Campos A, Sperandio F, Neto SC. Influência dos Achados Intra-operatórios no Resultado Anatômico das Miringoplastias. Arq Int Otorrinolaringol. 2006, 10(4):284288.

5. Cruz OLM, Costa SS, Kluwe LH, Smith MM. Timpanoplastias. In: Cruz OLM, Costa SS. Otologia Clínica e Cirúrgica. Rio de Janeiro: Revinter; 2000. p.245-70.

6. Porto G. Da cirurgia no tratamento das otites médicas supuradas crônicas. Rev Bras Otorrinolaringol. 1958, 26(5):15-25.

7. Albert Mudry, MD, PhD, Lausanne, Switzerland. History of myringoplasty and tympanoplasty type I. Otolaryngology-Head and Neck Surgery. 2008, 139:613614.

8. Bhat NA, Ranit De. Retrospective Analysis of Surgical Outcome, Symptom Changes, and Hearing Improvement Following Myringoplasty. J Otol. 2000, 29(4):229-32.

9. Sheehy JL et Anderson RG. Myringoplasty. A review of 472 cases. Ann Otol Rhinol Laryngol. 1980, 89(4 Pt 1):3314.

10. Kotecha B, Fowler S, Topham J. Myringoplasty: a prospective audit study. Clin Otolaryngol. 1999, 24(2):1269 .

11. Black JH, Wormald PJ. Myringoplasty-effects on hearing and contributing factors. S Afr Med J. 1995, 85(1):41-3.

12. Pinar E, Sadullahoglu K, Calli C, Oncel S. Evaluation of prognostic factors and middle ear risk index in tympanoplasty. Otolaryngology-Head and Neck Surgery. 2008, 139:386-390.

13. Emmett JR. Age as a factor in the success of tympanoplasty: A comparison of outcomes in the young and old. Am J Otol. 1996, 28:285-6.

14. Berger G, Berger S. Paediatric revision myringoplasty: outcomes and prospects. J Laryngol Otol. 2002, 116:6904.

15. Lin A, Messner A. Pediatric tympanoplasty: factors affecting success. Current Opinion in Otolaryngology \& Head and Neck Surgery. 2008, 16:64-68.

16. Sckolnick J, Mantle B, Li J, Chi D. Pediatric Myringoplasty: Factors That Affect Success-A Retrospective Study. Laryngoscope. 2008, 118:723-729.

17. Ribeiro J, Cerejeira R, Soares V, Gapo C, Romao J, Paiva A. Pediatric Tympanoplasties: Anatomical and Functional Results. Scientific Oral Presentations, p67.

18. Caye-Thomasen P, Nielsen T, Tos M. Bilateral Myringoplasty in Chronic Otitis Media. Laryngoscope. 2007, 117:903-906.

19. Gersdorff M, Garin P, Decat M, Juantegui M. Myringoplasty: long-term results in adults and children. Am J Otol. 1995, 16(4):215-8.

20. Sperling NM, Kay D. Diagnosis and Management of the Lateralized Tympanic Membrane. The Laryngoscope. 2000, 110(12):1987-1993.

21. Eby T. Prevention and Treatment of Tympanic Membrane Blunting. Middle ear mechanics in research and Otology. Proceedings of the 4th International Symposium. 2006, p:177-182.

22. Coifman H. Miringoplastia: Análise dos resultados cirúrgicos para avaliação de seu aprendizado em serviço universitário. Rev Bras Otorrinolaringol. 1992, 58(2):108112. 